Apidologie, 1973, 4 (3), 275-281.

\title{
DIE REPRÄSENTIERUNG DES CITRUS-POLLENS IN ITALIENISCHEN ORANGENHONIGEN
}

\section{L'abondance relative du pollen de Citrus dans les miels d'oranger italiens}

\author{
G. VORWOHL
}

Universität Hohenheim (LH) Landesanstalt für Bienenkunde

\author{
SUMMARY \\ THE REPRESENTATION OF GITRUS POLLEN IN \\ ITALIAN ORANGE HONEYS
}

By means of 61 honeys which had to be tested in routine investigations for their commercial qualification as orange honey we watched the coherence between the percentage of Citrus pollen and the absolute number of pollen grains. As expected for an underrepresented pollen, the absolute pollen contents at first decrease with increasing percentage of Citrus pollen. But in honeys with high percentages of Citrus pollen we also observe an augmentation of the absolute pollen contents. Different possibilities of explication are considered and the importance for practical examination of such curves is discussed.

\section{ZUSAMMENFASSUNG}

Anhand eines Materials von 61 Honigen, bei denen im Rahmen der Routineuntersuchung geprüft werden mußte, ob sie als Orangenhonig handelsfähig seien, wurde der Zusammenhang zwischen dem Prozentsatz an Citrus-Pollen und der absoluten Pollenzahl verfolgt. Zunächst nimmt, wie man das bei einem Pollen, der als unterrepräsentiert gilt, zu erwarten hat, mit steigendem Prozentsatz an Citrus-Pollen der absolute Pollengehalt ab. Bei Honigen mit hohen Prozentsätzen von Citrus-Pollen nimmt aber auch der absolute Pollengehalt wieder zu. Es werden verschiedene Erklärungsmöglichkeiten herangezogen und die Bedeutung solcher Kurven für die Beurteilungspraxis diskutiert. 


\section{EINLEITUNG}

Die Melissopalynologie hat sich - wie andere Zweige der Palynologie auch - mit Repräsentationsproblemen auseinanderzusetzen (MAURIzio 1939, Berner 1952, Haslinszky 1955, Pritsch 1956, Demianowicz 1961).

Aus dem Vorhandensein eines bestimmten Pollens im Honig ziehen wir den Schluß, daß die Bienen die Blüten der P flanzen besuchen, die diesen Pollen erzeugen. Diese Schlußfolgerung ist unproblematisch.

Aus der Häufigkeit eines bestimmten Pollens im |Honig ziehen wir Rückschlüsse auf den Anteil, den der Nektar der entsprechenden P flanze am Zustandekommen des Honigs hatte. Diese Interpretation ist problematischer. $20 \%$ eines Pollens $\mathrm{X}$ in einem Honig bedeutet nicht ohne weiteres, daß der Honig auch zu $20 \%$ aus dem Nektar $\mathrm{X}$ entstanden ist, der Nektaranteil kann größer oder kleiner sein.

Unsere Kenntnisse über die Repräsentierung bestimmter Pollen lassen sich erweitern und vertiefen :

a) Durch Untersuchung von Honigen, bei denen durch Feldbeobachtung gesichert ist, aus welchen Trachtquellen sie vorwiegend stammen und deren Gewinnungsart bekannt ist.

b) Durch Modellversuche (Einartenhonige, Demianowicz 1961).

c) Durch kritische Prüfung der bereits gewonnenen Regeln in der Untersuchungspraxis.

$\mathrm{Zu}$ diesem letzten Punkt soll der vorliegende Bericht beitragen : Im Jahre 1970 erhielten wir 92 Proben italienischer Honige zur Untersuchung. Es sollte geprüft werden, ob sie als Orangenhonig in den Handel gebracht werden können.

Dieses Material wurde benutzt, um den Zusammenhang zwischen dem Prozentsatz an Citrus-Pollen und der absoluten Pollenzahl herauszuarbeiten.

\section{MATERIAL UND METHODEN}

Untersucht wurden, wie schon erwähnt, 92 Proben süditalienischer Herkunft. Da es sich um Handelsproben handelt, fehlen nähere Angaben über den Ort, an dem der Honig erzeugt wurde und auch über die Citrus-Arten bzw. -Sorten, von denen der Honig stammt.

Die Proben wurden einer orientierenden Analyse unterzogen, ferner wurde die Pollenhäufigkeit geschätzt (s. Louveaux, MaUrizio und VorwoHL, 1970 a, b und c). Die Auszählung des absoluten Pollengehalts erfolgte mit Hilfe des Millipore-Filter-Verfahrens von Louveaux (1961). Gezählt wurden die Pollen insgesamt, ohne Differenzierung zwischen Citrus und den Pollen anderer nektarspendender oder nektarloser Arten. Pilzelemente und Algen wurden nicht gezählt, da sie im Immersionsöl manchmal schwer ansprechbar sind. 
Die Honigmenge reichte nicht bei allen 92 Proben für die absolute Zählung aus. Daher wurden die Restmengen von Honigen mit ähnlichem Pollenbild vereinigt. Insgesamt wurden 61 Proben der absoluten Zählung unterworfen.

\section{ERGEBNISSE}

Das Pollenspektrum der Honige war recht einheitlich. Sehr häufig fanden sich entweder Citrus oder Hedysarum coronarium (= Sulla). Häufig kamen neben Citrus und Sulla vor : Lotus $(6 x)$, ein Lotus- ähnlicher LeguminosenPollen ( $4 x$ ), Myrtaceen $(4 x)$, Castanea sativa $(4 x)$ und Trifolium repens-

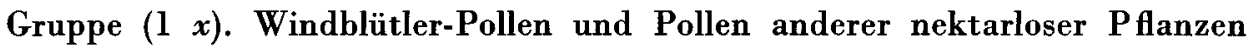
waren selten, desgleichen Pilzelemente und Algen.

Das pauschale Auswertungsverfahren, das nur die absolute Pollenzahl berücksichtig ohne Aufschlüsselung nach Pollenarten, ist also vertretbar. Durch das häufige Auftreten (15-45\%) von Castanea wurden die absoluten Pollenzahlen nicht merklich beein flußt. Die Honige mit Castanea liegen mit ihren Zahlen nicht auffällig höher. Insgesamt liegen alle Zählungen recht niedrig. Der höchste absolute Pollengehalt erreicht knapp 25000 PK/10 g Honig.

Die Resultate der absoluten Pollenzählung wurden graphisch zu den Prozentsätzen an Citrus-Pollen in Beziehung gesetzt (Abb. 1). Auf der Ordinate findet sich die Anzahl der Pollenkörner pro $10 \mathrm{~g}$ Honig; auf der Abszisse die Prozentsätze der gefundenen Citrus-Pollen. Wegen der besseren Übersichtlichkeit und wegen der bekannten großen Fehlerbreite bei der Schätzung der Pollenhäufigkeit (Vergeron, 1964) wurden die Prozentzahlen zu Klassen zusammengefaßt (Kl. 1 : $0-10 \%$ Citrus-Pollen, Kl. II : $11-20 \%$, usw.). Neben den Einzeldaten sind die Durchschnittswerte der absoluten Zählung durch starke Punkte hervorgehoben.

Mit steigendem Prozentsatz an Citrus-Pollen ist zunächst ein Fallen der absoluten Pollenzahl zu beobachten. Das ist zu erwarten : Je größer der Prozentsatz an Citrus-Pollen, desto größer der Prozentsatz an Citrus-Nektar. Da Citrus die Tendenz hat, wenig Pollen und entsprechend pollenarmen Nektar zu erzeugen, muß mit dem Wachsen des pollenarmen Citrus-Nektar-Anteils am Honig auch die absolute Pollenzahl sinken. Merkwürdigerweise liegt aber die absolute Pollenzahl bei hohen Prozentsätzen von Citrus-Pollen wieder höher (durchschnittlich $20210 \mathrm{PK}$ in Honigen mit mehr als $60 \%$ Citrus-Pollen). In der Probengruppe mit 50-60\% Citrus-Pollen finden wir zwischen 6 130$23400 \mathrm{PK}$ in $10 \mathrm{~g}$ Honig, also sehr divergente Werte.

Wir haben demnach bei Citrus-Honigen aus dem gleichen Herkunftsgebiet Proben mit normalem absolutem Pollengehalt und hohen Prozentsätzen von Citrus-Pollen. Hier liegt offensichtlich eine normale Repräsentation vor. Daneben gibt es ebenso offensichtlich Citrus-Honige mit niedriger absoluter 


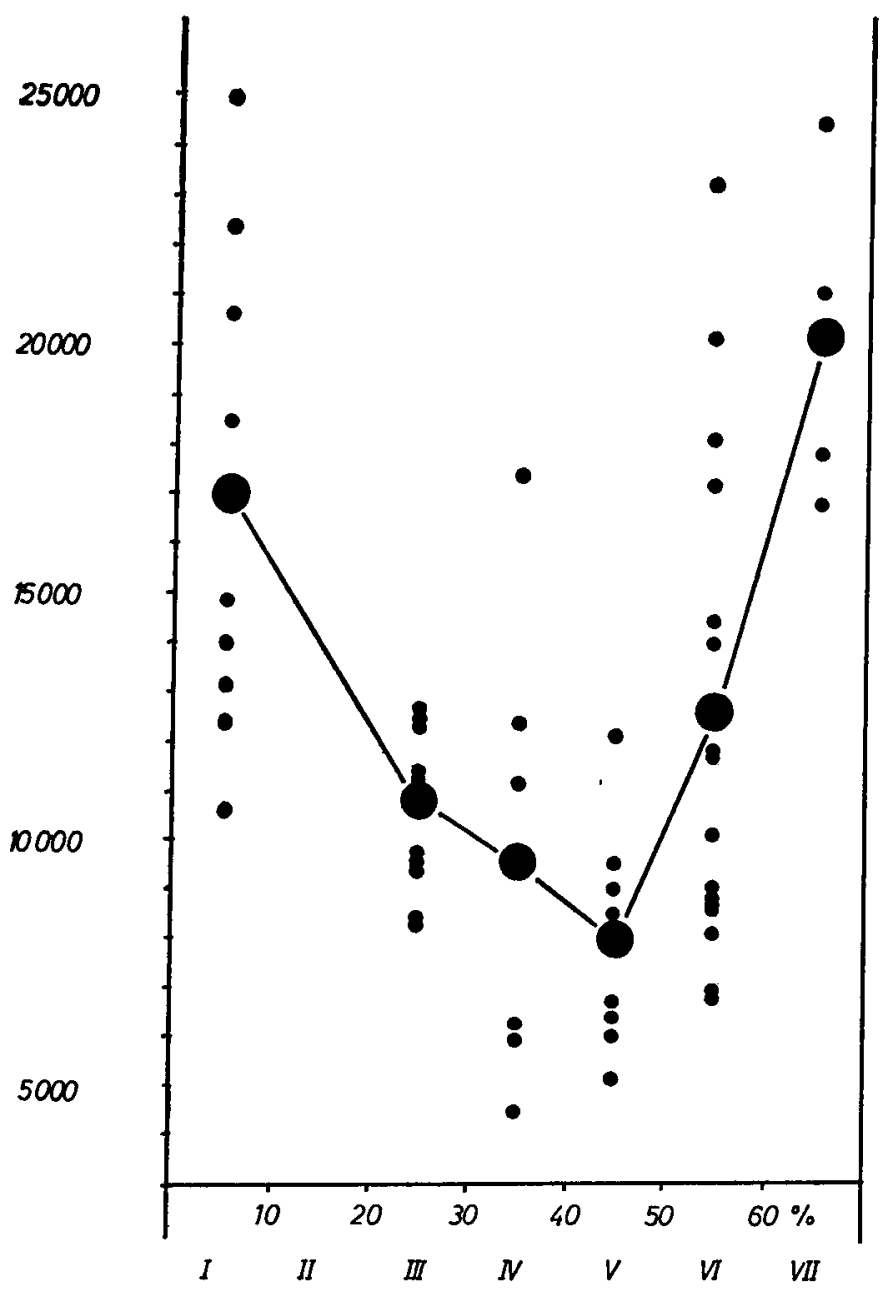

Aвв. 1 - Beziehung zwischen der absoluten Pollenzahl und dem Prozentsatz an Citrus-Pollen.

Ordinate : absolute Pollenzahl in $10 \mathrm{~g}$ Honig.

Abszisse : Prozentsatz Citrus-Pollen und Klasseneinteilung.

Die kleinen Punkte stellen die Einzelwerte dar. Die dicken

Punkte die Durchschnittswerte für eine Klasse.

FIc. 1 - Relation entre le nombre absolu de grains de pollen et le pourcentage de pollen de Citrus.

En ordonnées : nombre absolu de grains de pollen dans $10 \mathrm{~g}$ de miel. En abscisses : pourcentage de pollen de Citrus et répartition dans les classes d'abondance. Les points les plus petits se rapportent aux valeurs individuelles; les points les plus gros représentent les valeurs moyennes pour une classe.

Pollenzahl und geringen bis mittleren Prozentsätzen von Citrus-Pollen. In diesen Fällen erscheint der Citrus-Pollen unterrepräsentiert. Als Erklärungsmöglichkeiten für dieses Phänomen können wir heranziehen :

1. Unterschiedliche Pollenproduktion durch verschiedene Citrus-Varietäten, worauf schon MAURIzio (1958) hinwies, mit entsprechend wechselnder “ primärer Einstäubung 》 (DEMIaNowicz). 
2. Unterschiedliche " sekundäre Einstäubung " des Honigs im Bienenstock. Die Bienen tragen Pollen unmittelbar zur Ernährung der Brut und der Jungbienen ein. Von diesem Pollen können kleine Mengen in den separat gelagerten Honig geraten (Louveaux, 1958).

3. Unterschiedliche “ tertiäre Einstäubung 》 bei der Honiggewinnung. Der von den Bienen direkt gesammelte Pollen wird als sogenanntes Bienenbrot in den Zellen aufbewahrt. Beim Schleudern des Honigs bleibt er an seinem Platz. Es besteht aber die Möglichkeit, daß beim Entdeckeln der Waben Bienenbrot angekratzt wird und kleine Mengen in den Honig geraten. Je nach Beutentyp und Wirtschaftsweise der Imker können die Honigwaben viel oder wenig Bienenbrot enthalten. Entsprechend ist auch die tertiäre Einstäubung keine feste Größe.

Wie mir Dr. Bretschko, Bir Bou Rekba, Tunesien, und Dr. Barbier, Marokko, mitteilten, höseln die Bienen nur wenig Citrus-Pollen. Entsprechend dürfte sich auch die sekundäre und tertiäre Einstäubung im allgemeinen in Grenzen halten. Man kann die Möglichkeit einer Veränderung des Pollenspektrums durch 2. und 3. jedoch nicht von vorneherein in Abrede stellen.

Wie schon erwähnt, handelt es sich bei dem untersuchten Material um Handelsproben. Freilandbeobachtungen an den Trachtquellen, Angaben über die imkerliche Betriebsweise und über Details der Honiggewinnung liegen nicht vor. Es kann also nicht entschieden werden, inwieweit primäre, sekundäre und tertiäre Einstäubung für die beobachteten Phänomene verantwortlich ist.

Bisher hat man im allgemeinen nur die unterschiedliche primäre Einstäubung bei der Beurteilung von Pollenspektren herangezogen. Es ist aber nicht uninteressant, die Ergebnisse der vorliegenden Untersuchung unter dem Gesichtspunkt der sekundären und tertiären Einstäubung zu interpretieren. Die Honige des “ Mittelfelds » von Abb. 1 (Klassen III-V) würden im Lichte dieses Erklärungsversuches ihren Pollengehalt im wesentlichen der “ primären Einstäubung 》 verdanken, also dem Pollengehalt des Citrus-Nektars. Die höhere absolute Pollenzahl und der höhere Citrus-Prozentsatz der Honige in Klasse VII und der pollenreicheren Honige der Klasse VI wären auf eine stärkere « sekundäre und/oder tertiäre Einstäubung » mit Citrus-Pollen zurückzuführen.

Schließlich könnte man bei einigen der Honige in Klasse I annehmen, daß ihr Pollengehalt zu einem größeren Teil auf sekundärer und /oder tertiärer Einstäubung mit Pollen beruht, die neben Citrus eingetragen wurden, wobei allerdings vorauszusetzen wäre, daß der Citrus-Nektar sehr pollenarm ist und die Bienen keinen Citrus-Pollen direkt eintragen.

In jedem Fall dürfte es nützlich sein, solche Zusammenstellungen für Sortenhonige aller Art anzulegen, wobei auch die verschiedenen Herkunftsge- 
biete zu berücksichtigen wären, also im Falle von Citrus-Honigen Californien, Florida, Spanien, Türkei usw. Solche Repräsentationskurven erleichtern die Interpretation späterer Analysen, da sie Durchschnittswerte und Streuungen auf einen Blick zeigen. Wertkombinationen wie die Klassen VII und VI der vorliegenden Darstellung lassen mit großer Sicherheit den Schluß zu, daß es sich um Citrus-Honig handelt. Auch wenn man die hohen absoluten Pollenzahlen auf sekundäre oder tertiäre Einstäubung zurückführen will, so zeigt doch der Befund, daß Citrus stark beflogen wurde und kaum andere Trachten zum Honig beigetragen haben. Eine Irrtumsmöglichkeit wäre allerdings denkbar, nämlich daß sekundäre oder tertiär eingearbeiteter Citrus-Pollen andere extrem pollenarme Nektare kaschiert, z.B. Lavendel und Lavandin, was aber eine wenig wahrscheinliche Kombination ist.

Wertkombinationen wie in den Klassen III, IV und V sind sozusagen ideal, es finden sich hinreichende Citrus-Pollen-Prozentsätze bei niedriger absoluter Pollenzahl. Hier wird man auf weitere Verfahren zum Beweis der Herkunft verzichten können, solche wären nur bei « Ausreißern » angebracht, wie z.B. bei der Kombination IV/17 420. Wertkombinationen wie in KI. I sind auch bei Citrus-Honigen denkbar, wenn man extreme Unterrepräsentierung des Citrus-Pollens annimmt und bei höherer absoluter Pollenzahl Überrepräsentierung der begleitenden Pollen. In unserem Material tritt Citrus besonders in Partnerschaft mit Sulla auf. $\mathrm{Ob}$ diese als überrepräsentiert gelten darf, ist noch nicht ausreichend geklärt. Auf jeden Fall dürfen Honige mit Wertkombinationen aus der Kl. I nur dann als Citrus-Honige bezeichnet werden, wenn dafür neben dem Sinnenbefund auch noch andere Anhaltspunkte vorhanden sind, wobei besonders an den Nachweis des Methylanthranilats auf chemischen Wege oder durch Gaschromatographie zu denken ist.

Die vorliegende Untersuchung wirft Fragen auf, die nur an Ort und Stelle, d.h. in einem Gebiet mit Citrus-Plantagen gelöst werden können. Interessant wäre vor allem, die quantitative Untersuchung der Pollenernte der Bienenvölker während der Citrus-Blüte. Dabei müßten die verschiedenen Varietäten und ihre unterschiedliche Produktion besonders berücksichtigt werden. Vom Ergebnis dieser Untersuchung her ließe sich die Frage beantworten, ob eine nennenswerte Beimengung von Citrus-Pollen zum Honig auf sekundärem oder tertiärem Wege überhaupt möglich ist.

Eingegangen im Februar 1973. Reçu pour publication en février 1973.

\section{DANKSAGUNG}

Frau D. Friedl und Frl. U. Krukenberg danke ich für die Durchführung der langwierigen Zählarbeit.

Das Programm « Vergleichende palynologische Untersuchung der Honige des Mittelmeergebiets $)$, zu dem das hier behandelte Teilproblem gehört, wird mit Förderung der Deutschen Forschungsgemeinschaft durchgeführt, wofür ich gleichfalls zu danken habe. 


\section{RESUME}

Dans le cadre des analyses de routine destinées à préciser la valeur de l'appellation commerciale " miel d'oranger ", 61 échantillons de miel ont été étudiés en vue de déterminer la relation existant entre le pourcentage de pollen de Citrus et le nombre absolu de grains de pollen par unité de poids de miel. Comme il est normal pour un pollen qui est réputé sousreprésenté, on constate tout d'abord que plus le miel est pauvre en pollen, plus le pollen de Citrus représente un pourcentage élevé du total. Cependant, pour les miels qui présentent un fort pourcentage de pollen de Citrus on constate que la teneur absolue en pollen augmente également. On présente différentes explications possibles de ce phénomène et l'intérêt pratique de la courbe obtenue est discuté.

\section{LITERATUR}

-

Berner U., 1952. Die Auswertung der Pollenanalyse. Arch. Bienenkde. 29, 33-38.

Demianowicz Zofia, 1961. Pollenkoeffizienten als Grundlage der quantitativen Pollenanalyse des Honigs. Pszczelnicze Zeszyty Naukowe 5, (2), 95-107.

Hazslinszky B., 1955. Pollenanalyse und Auswertung des Pollenspektrums in Honig. Elelmszervizgalati Közl 1, (1), 3-18.

Louveaux J., 1958. Recherches sur l'origine dans le miel du pollen de plantes entomophiles dépourvue de nectaires. Ann. Abeille 2, 89-92.

Louveaux J., 1961. Techniques améliorées pour l'analyse pollinique des miels. Z. Bienenforsch. 5, (7), 199-204.

Louveaux J., Maurizio Anna and Vorwohl G., 1970 a. Methods of melissopalynology. Bee World 51, (3), 125-138.

Louveaux J., Maurizio Anna and Vonwohl G., 1970 b. Methodik der Melissopalynologie. Apidologie 1, (2), 193-209.

Louveaux J., Maurizio Anna et Vorwohl G., 1970 c. Les méthodes de la Mélisso-Palynologie. Apidologie 1, (2), 211-227.

MaUnizio Anna, 1939, Untersuchungen zur quantitativen Pollenanalyse des Honigs. Mitt. Lebensmitt. unters. Hyg. 30, (1/2), 27-69.

Maurizio Anna, 1949. Pollenanalytische Untersuchungen an Honig und Pollenhöschen. Bh. Schweiz. Bien. z., 2, (18), 320-455.

Maurizio Anna, 1958. Beiträge zur quantitativen Pollenanalyse des Honigs, 3. Absoluter Gehalt p flanzlicher Bestandteile in Esparsette, Luzerne, Orangen- und Rapshonigen. Ann. Abeille 2, 93-106.

Pritscr G., 1956. Zum Problem der mikroskopischen Pollenanalyse des Bienenhonigs. Wiss. Z. Humboldt-Univ. Berlin, Math. natwiss. Reihe 6, (2), 197-204.

VERGERON P., 1964. Interprétation statistique des résultats en matière d'analyse pollinique des miels. Ann. Abeille 7, (4), 349-364.

Anschrift des Verfassers : Dr. G. Vorwohl, Universität Hohenheim, Landesanstalt für Bienenkunde, D 7 Stuttgart 70, Emil-Wolff-Str. 60, BRD. 